Check for updates

Cite this: RSC Adv., 2017, 7, 41603

\title{
Light filter tailoring - the impact of light emitting diode irradiation on the morphology and optical properties of silver nanoparticles within polyethylenimine thin films
}

\author{
F. Kemper, (D) *ab E. Beckert, ${ }^{a}$ R. Eberhardt ${ }^{a}$ and A. Tünnermann ${ }^{a b}$
}

In this contribution in situ emission filter generation for, e.g. fluorescence light detection by morphology tailoring of silver nanoparticles within a polymer layer, is presented for the first time. After depositing a pretreated film of polyethylenimine containing spherical silver nanoparticles the composite layer was irradiated with green LEDs (peak wavelength $530 \mathrm{~nm}$ ). This leads to a morphology change of the silver nanoparticles and therefore to a change in absorption properties of the resulting polymer film with an absorption maximum at $550 \mathrm{~nm}$. This tailoring of silver nanoparticles embedded in a transparent polymer matrix could address many novel applications such as adaptable light filters for future lab-on-a-chip applications. A LED for fluorescence light excitation was used to illuminate the silver nanoparticles, which led to an increasing absorption of that particular light spectrum, and therefore in situ produced a fluorescence emission filter. The effect of the treatment time, the silver ion concentration, and the temperature at which the "light-shaping" was done was studied. The silver nanoparticles were investigated using UV/Vis-spectroscopy and scanning electron microscopy. The observed nanoparticle shapes give reasons for a novel hypothesis of nanoparticle evolution. The forced plasmon oscillation seems to cause excited regions at the silver nanoparticles where adsorbed silver ions are preferably photoreduced. This results in the possibility of tailoring the morphology and therefore the optical properties of the growing nanoparticles.

Received 27th July 2017

Accepted 21st August 2017

DOI: $10.1039 / c 7 r a 08293 b$

rsc.li/rsc-advances

\section{Introduction}

Because of their unique properties, silver nanoparticles (AgNPs) have become a valuable material for many applications. Conductive inks ${ }^{1}$ for electric interconnects ${ }^{2}$ and MEMS $^{3}$ utilize their high electric conductivity and low sintering temperatures; ${ }^{4}$ different sensing principles like surface enhanced Raman scattering (SERS), ${ }^{5}$ surface enhanced fluorescence, ${ }^{6}$ surface plasmon resonance (SPR) spectroscopy ${ }^{7}$ as well as numerous biomedical applications $^{8}$ utilize the unique optical properties of AgNPs. The physical and chemical properties of AgNPs highly depend on their size and morphology. ${ }^{9}$ To address all the mentioned applications by controlling the morphology and size of nanoparticles many synthesis routines have been investigated.

In general, AgNPs can be synthesized using physical, ${ }^{\mathbf{1 0}-13}$ chemical, ${ }^{14-16}$ photochemical, ${ }^{17-20}$ or biological methods. ${ }^{21-24}$ Chemical and photochemical reduction methods have been demonstrated to be the most appropriate to control the

${ }^{a}$ Fraunhofer Institute for Applied Optics and Precision Engineering (IOF), Albert-Einstein-Str. 7, D-07745 Jena, Germany.E-mail:falk.kemper@iof.fraunhofer.de ${ }^{b}$ Institute of Applied Physics, Abbe Center of Photonics (ACP), Friedrich Schiller University Jena, Max-Wien-Platz 1, D-07743 Jena, Germany morphology and size of AgNPs. In this contribution photochemical synthesis is used to generate the AgNPs. It can be split into two main steps including the reduction of $\mathrm{Ag}$ salts like $\mathrm{Ag}$ nitrate $\left(\mathrm{AgNO}_{3}\right)$ using a photo initiator, ${ }^{25,26}$ and preventing the forming AgNPs from agglomeration by adding a stabilizing agent. ${ }^{27}$ In the literature a variety of different stabilizing agents such as citrate, gallic acid, sodium dodecyl sulfate, ${ }^{28}$ and many polymers like poly(vinyl alcohol), polyethylene glycol, polyvinylpyrrolidone, poly(methyl methacrylate), and polyethylenimine $^{29-31}$ (PEI) were investigated.

All these reduction and shaping methods are performed using a solvent as supporting matrix. Only a few methods report of solid polymers as a matrix to produce and stabilize AgNPs. ${ }^{18,32-35}$ In these cases the resulting nanoparticles appeared to be spherical with an absorption peak at $\approx 400 \mathrm{~nm}$. Although morphology adapted AgNPs embedded within polymer layers could address a variety of applications there is no report about tailoring the nanoparticle morphology within a polymer layer, yet.

In this contribution we present a novel routine to grow and reshape AgNPs inside a PEI layer and thus tailor their absorption characteristics. This enables novel applications like in situ production of the emission filter in a lab-on-a-chip (LoC) 
fluorescence light detection setup. ${ }^{36}$ The emission filter is necessary to shield the detector from excitation light. In the past different light filter concepts for integration into LoC systems have been studied such as polarization filters, ${ }^{37}$ colorant filters, ${ }^{38,39}$ interference filters, ${ }^{39}$ and liquid crystal filters. ${ }^{40}$ All these filter concepts have different advantages and disadvantages. Colorant filters for example have a high transmission $(>95 \%)$ in their transmission band compared to polarization filters $(<50 \%)$. Their drawback is their potential use in only one special application and therefore the application dependent choice of specific colorants. By introducing silver nanoparticles as absorbing media this contribution presents a novel routine to overcome this issue. The surface plasmon resonance of the nanoparticles can be shifted within the visible spectrum, tailored to specific light absorption properties, and therefore replace many different colorants and production methods with one simple in situ tailoring.

\section{Novel light filter tailoring within LoC}

Fluorescent light detectors include a light source to excite the fluorescent light, a photodetector to detect the fluorescent light, and filters to separate the fluorescent light signal from the excitation light signal. To be cost efficient such setups can be constructed using fully solution processed organic light emitting electrochemical cells and fully solution processed organic photodiodes (OPD). ${ }^{36}$ In order to not joining them separately but directly integrating them onto a LoC the light filters have to be solution processed, too. Only colorant filters are reported to be solution processable so far. However, with their fixed light absorption properties they severely limit the potential versatility of a broad spectrum absorbing OPD to very few fluorescent dyes. Tailorable light absorption properties of solution processable layers would dramatically increase the variety of usable fluorescent dyes. Furthermore, they enable the use of the whole spectral potential of the OPD. Therefore they could provide a very cost efficient solution for integrating an OPD with emission filter onto LoC with addressing many different applications. The emission filter should absorb the excitation light. A smart way to tailor the filter properties would be to use the excitation light source to make the filter absorbing exactly its light spectrum. Fig. 1 shows a scheme of how the light tailoring of filters could be used within fluorescent light detectors.
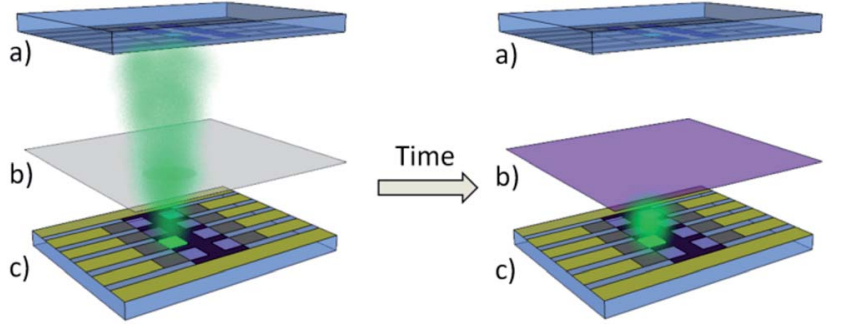

Fig. 1 Fluorescent sensor for LoC comprising a light detector (a), an emission filter (b) and a light source (c). By illuminating the transparent filter it can be tailored to absorb exactly the spectrum of the light illumination.
As we showed earlier the morphology and therefore the absorption properties of silver nanoparticles in water can be tailored by illuminating them with VIS-light. ${ }^{\mathbf{4 1}}$ The possibility of affecting their properties within polymer layers is discussed in this contribution.

\section{Experimental}

\section{Materials and characterization}

Silver nitrate (99.99\%), 2-hydroxy-4'-(2-hydroxyethoxy)-2methylpropiophenone (I2959) (98\%), sodium citrate dihydrate (99\%) and branched polyethyleneimine (PEI) were purchased from Sigma Aldrich. Ethanol was used as solvent for ink preparation and deionized water was used for preparing the suspensions for SEM sample preparation. All chemicals and solvents used in this work were of analytical grade and used without further purification. The layer thicknesses of the deposited layers were measured using a Zygo 600N white light interferometer. Absorption spectra of the inks and PEI thin films were recorded on the PerkinElmer UV-Vis spectrometer Lambda 950 at room temperature in air. Baseline correction was performed prior to all measurements. The nanoparticle shape was characterized using a Zeiss SEM. The nanoparticle generation and light treatment were done using a Hönle AG bluepoint 4 UV-lamp and green Cree high power LEDs.

\section{Solution and layer preparation}

Four seed solutions with different Ag contents were prepared out of an ethanol stock solution containing $20 \mathrm{mg} \mathrm{ml}^{-1}$ silver nitrate, $10 \mathrm{mg} \mathrm{ml}^{-1} \mathrm{I} 2959$ and $50 \mathrm{mg} \mathrm{ml}^{-1}$ PEI. To increase the solubility of silver nitrate and I2959 in the ethanol solution the PEI had to be dissolved first. The solution was ultra-sonicated for $30 \mathrm{~min}$ and then stirred for another $30 \mathrm{~min}$ at room temperature. The solution was diluted with $50 \mathrm{mg} \mathrm{ml}^{-1}$ of PEI in ethanol to reduce the $\mathrm{Ag}$ content, resulting in solutions containing $5 \mathrm{mg} \mathrm{ml}^{-1}, 10 \mathrm{mg} \mathrm{ml}^{-1}, 15 \mathrm{mg} \mathrm{ml}^{-1}$, and $20 \mathrm{mg} \mathrm{ml}^{-1}$ silver nitrate. All solutions were illuminated with $14 \mathrm{~W} \mathrm{~cm}^{-2}$ UV-light (bluepoint 4) for $40 \mathrm{~s}$ to generate spherical nanoparticle seeds, which should grow and reshape while illuminating them with VIS-light.

The resulting pretreated seed inks were spin coated in air on microscope glass slides using an Ossila desktop spin coater at $500 \mathrm{rpm}$ for $60 \mathrm{~s}$. For better surface wetting and homogeneity the glass slides were cleaned and plasma treated before the deposition. To remove the solvent from the as deposited layers the samples were vacuum dried for $10 \mathrm{~min}$. The thickness of the dried layers was measured with a white light interferometer and determined to be $500 \mathrm{~nm}$. Afterwards, the samples were stored in nitrogen atmosphere to prevent the AgNPs from oxidation.

All samples were treated in air at room temperature or $70^{\circ} \mathrm{C}$ in a self-made reaction chamber using two green high-power Cree LEDs with a peak emission at $530 \mathrm{~nm}$. The LEDs were operated at $200 \mathrm{~mA}$ for different time periods. For spectra comparison and SEM sample preparation water based seed solutions containing $1 \mathrm{mM} \mathrm{Ag}, 1 \mathrm{mM}$ I2959 and $5 \mathrm{mM}$ sodium citrate dihydrate were also light treated at room temperature. To 
prepare the samples for the SEM observation the treated suspensions were drop-casted and dried on cleaned silicon wafers. The optical spectra of all samples were measured in the range of $320 \mathrm{~nm}$ to $860 \mathrm{~nm}$.

\section{Results and discussion}

A solution of PEI, $\mathrm{AgNO}_{3}$ and 2-hydroxy-4'-(2-hydroxyethoxy)-2methylpropiophenone (I-2959) in ethanol was UV-irradiated to generate silver seeds. The resulting solution was spin-coated on glass to deposit AgNPs containing PEI layers. Following a photochemical approach the morphology and size of AgNPs can be tailored by LED light illumination. ${ }^{25}$ Therefore the PEI layers were irradiated with green LEDs (peak wavelength 530 $\mathrm{nm}$ ) to change the AgNPs morphology (the experimental details are reported in the Experimental section).

To investigate the nanoparticle evolution and to identify the morphology of the AgNPs SEM-imaging has to be used. However, since the AgNPs within the PEI layers are embedded in a nonconductive matrix sufficient SEM-imaging is impossible. Furthermore, dissolving the coloured PEI-layers in ethanol or water led to an oxidation and decomposition of the AgNPs. Therefore, AgNP suspensions in water were prepared to enable this observation. By comparing the optical spectra of the treated suspensions and the PEI layers conclusions about the AgNP morphology within these layers are possible.

\section{Optical characterization of AgNP suspensions in water}

Fig. 2 shows the resulting spectra and a photograph of AgNPs suspension samples in water with different treatment times (increasing from left to right) by green light irradiation.
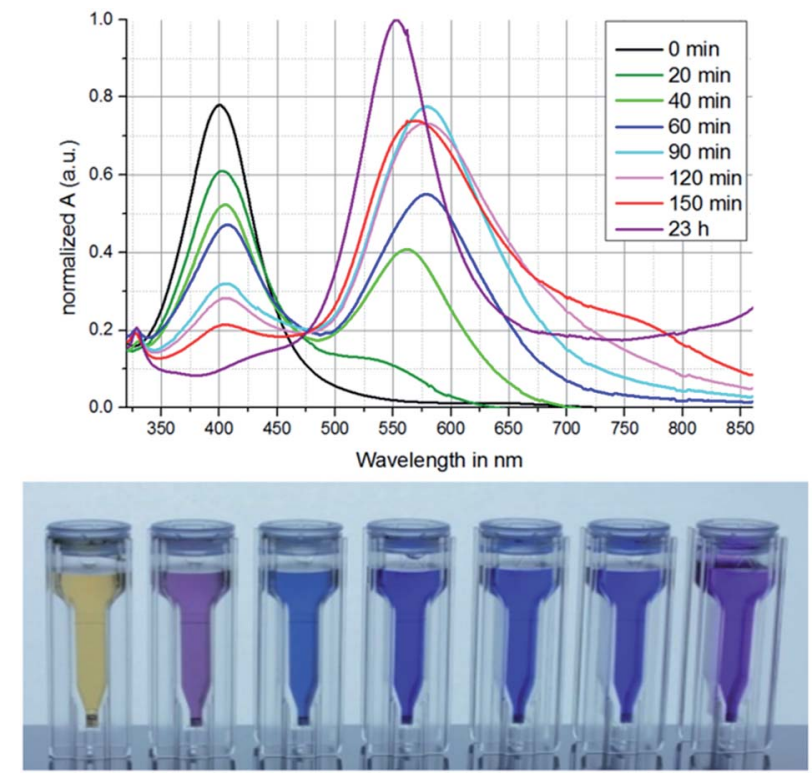

Fig. 2 Absorption spectra and corresponding samples of AgNPsuspensions (increasing irradiation time from left to right) illuminated with green light in the range of $20 \mathrm{~min}$ to $23 \mathrm{~h}$.
In advance all samples were treated with UV light to generate spherical AgNP seeds. The seed sample (0 min) shows a large absorption peak at $\approx 400 \mathrm{~nm}$. This indicates the formation of spherical AgNPs. ${ }^{25}$ With increasing time of green light irradiation the peak at $400 \mathrm{~nm}$ decreases and an absorption peak at $\approx 560 \mathrm{~nm}$ increases after $20 \mathrm{~min}$. Until a treatment duration of 120 min the absorption peak shifts to $\approx 575 \mathrm{~nm}$ and then back to $\approx 550 \mathrm{~nm}$ for $23 \mathrm{~h}$ of irradiation time. This indicates a nanoparticle evolution initiated by the green light exposure. The number of spherical shaped AgNPs decreases leading to the decrease of the peak at $\approx 400 \mathrm{~nm}$ whereas the number of differently shaped AgNPs increases. ${ }^{25}$ That means that the nanoparticles start to reshape while irradiation. During this evolution the AgNPs pass different morphologies which at the same time lead to changes in the absorption signal. The reshaping can only occur when the AgNPs grow at the same time. The whole process can be explained by excited regions at the AgNPs because of the forced plasmon oscillation during the green light irradiation. At these excited positions adsorbed Agions get photoreduced, resulting in nanoparticle growth to a certain shape. ${ }^{26}$ The impact of irradiation treatment is also observable by a color change of the suspensions as shown in Fig. 2b. After green light irradiation the colour changes from yellow to purple and blue with increasing treatment time. The experiment was stopped after $23 \mathrm{~h}$ because the nanoparticles started to agglomerate (not shown here).

\section{SEM characterization of AgNPs}

To investigate the nanoparticle evolution during the light treatment we chose a sample with both peaks at $400 \mathrm{~nm}$ and $\approx 550 \mathrm{~nm}$ corresponding to different evolution states. The sample treated for $150 \mathrm{~min}$ is the most appropriated for this purpose, since the evolution of the nanoparticles should be the most advanced without being completed. Within this sample many differently shaped nanoparticles were found (see Fig. 3). Besides small spherical nanoparticles there are circular plates, irregular hexagonal plates, regular hexagonal plates, and triangular plates. Fig. $3 \mathrm{~h}$ shows the ratio of the different shapes found in the sample. Stamplecoskie et $a .^{25}$ describe a conversion of all nanoparticles from spherical shapes to differently shaped nanoplates. With $505 \mathrm{~nm}$ illumination they produced hexagonal nanoplates and with 590/627 nm illumination triangular nanoplates were grown. However, our findings indicate that not all nanoparticles start shaping at the same time. Furthermore, our chosen emission peak of the LED at $530 \mathrm{~nm}$ seems to promote the formation of hexagonal plates and triangular plates. The surface plasmon resonance of these different shaped nanoplates highly depends on their size and final morphology. Therefore, hexagonal nanoplates ${ }^{25}$ as well as triangular nanoplates ${ }^{42}$ can show comparable absorption spectra.

For explaining nanoplates of different sizes within our sample we suggest a novel evolution route of hexagonal and triangular nanoplates under constant LED irradiation based on the work of Maillard et al. ${ }^{26}$ and Stamplecoskie et al. ${ }^{25}$ 


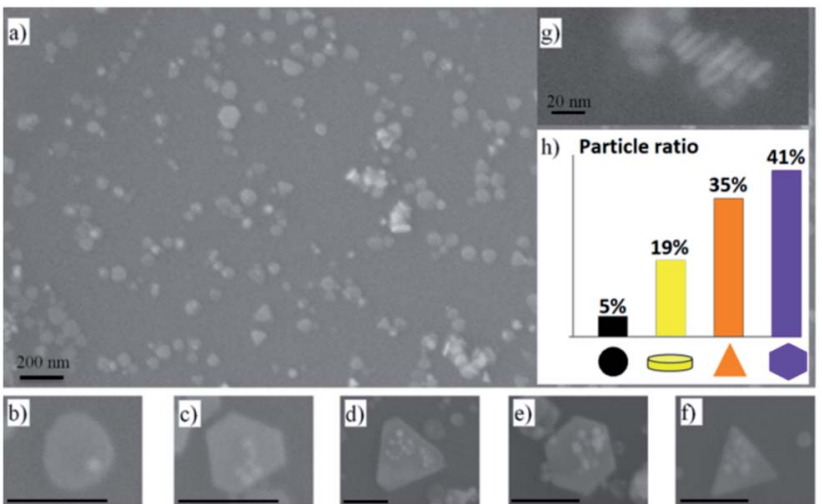

Fig. 3 SEM images of the produced silver nanoparticles $(a-g)$ and the percentage distribution of all found shapes (h). Different sizes and shapes including very small spherical particles (in a), circular plates (b), regular (e) and irregular (c and d) hexagonal plates, and triangular plates (f) were found. The thickness of all these plates is the same (g), indicating nanoparticle growth only in plane direction. The scale bar of the single nanoparticles indicates $100 \mathrm{~nm}$.

The general evolution process can be split into three different phases (Fig. 4a). At first, spherical AgNPs form under UV-light irradiation. ${ }^{25}$ In a second step circular disc-like nanoplates form because of the irradiation with VIS-light. ${ }^{26}$ Fig. $3 \mathrm{~g}$ shows that within our sample nanoplates with different diameters have approximately the same thicknesses. This indicates that at a certain point the particle growth only occurs in plane direction. In a third step these disc-like nanoplates grow resulting in different morphologies. Maillard et al. ${ }^{\mathbf{2 6}}$ explained the growth mechanism with excited regions at the nanoparticles, where adsorbed Ag-ions get reduced. They assigned a key role to citrate. In their opinion the nanoplates grow in disc-like shapes until irregularities occur. Because of the formation of these irregularities other nanoparticle shapes start to form. We believe that in our case small disc-like particles grow to small triangular nanoplates. After finishing the triangular shape the nanoparticles will grow forming irregular hexagonal shapes, passing regular hexagons resulting in larger triangles again (see Fig. 4b). Therefore, all "evolution states" in many different sizes can be found within our sample (see Fig. $3 \mathrm{~h}$ ). To further verify this theory effective triangular shapes of hexagonal and triangular nanoplates were compared. Therefore, the long sides of the hexagons were extended until they overlapped. Fig. 5a shows triangular and hexagonal

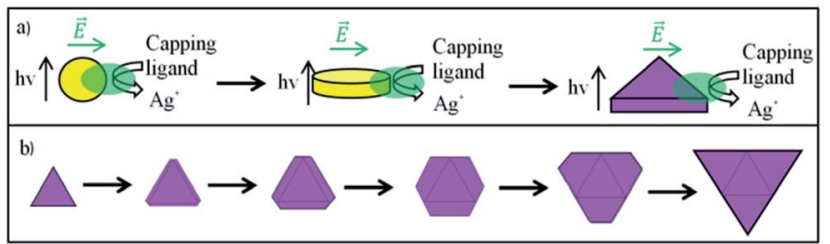

Fig. 4 (a) Nanoparticle evolution from spherical nanoparticles to triangles. (b) Triangular nanoparticles grow to irregular hexagonal nanoplates, further on to regular hexagonal nanoplates, and finally to triangular nanoplates again.
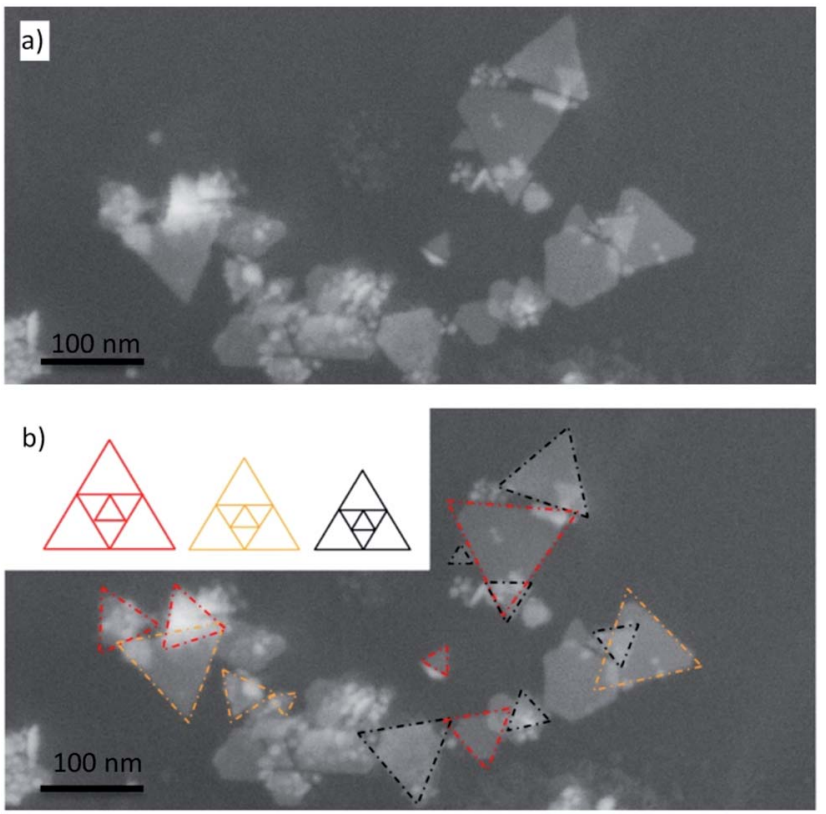

Fig. 5 SEM images of the produced silver nanoparticles (a) and their effective triangular areas highlighted with differently coloured frames (b). The different frame colours correspond to triangular/hexagonal nanoparticles with a size relation shown in the sketch.

nanoparticles found in our sample and Fig. 5b the same particles with their effective triangular profiles indicated by the different coloured frames. The different frame colours correspond to different sizes of the disc-like nanoplates, at which the irregularities formed. By comparing these triangular frames between particles highlighted with the same frame colour the predicted size relation of the evolution theory was confirmed (compare Fig. $4 \mathrm{~b}$ and $5 \mathrm{~b}$ ). The biggest nanoparticles show exactly four times the area of the medium sized nanoparticles which show exactly four times the area of the smallest nanoparticles. Therefore, it can be assumed that small triangles grow to hexagons and larger triangles as described before and in Fig. 4.

\section{AgNP shaping in PEI thin films}

Within PEI thin films including embedded Ag-nanoparticles we also observed a change of the absorption spectra caused by green LED light irradiation. Absorption peaks comparable to the peaks in water were found indicating the same nanoparticle reshaping within these films as well. As mentioned before, Maillard et al. ${ }^{\mathbf{2 6}}$ assign a key role for the reshaping to citrate. However, in the shown case no citrate is present, proving that PEI can also act as a capping ligand and a photoreducing agent for sufficient nanoparticle shaping. In addition, depending on the temperature and the Ag-precursor concentration within the PEI the resulting absorption spectra tremendously deviated from each other. Our findings are discussed in the next chapters.

\section{Temperature dependency}

The temperature effect on the nanoparticle evolution in the PEIlayers is exemplarily discussed for the samples deposited from 
a solution containing $5 \mathrm{mg} \mathrm{ml}^{-1}$ silver nitrate. They were irradiated at room temperature or at $70{ }^{\circ} \mathrm{C}$ for different time periods. The $0 \mathrm{~h}$ sample indicates the PEI-sample before treatment.

For comparison, samples without illumination were heated to $70{ }^{\circ} \mathrm{C}$ as well. For the non-irradiated samples there is a prominent increase of the absorption peak at $\approx 420 \mathrm{~nm}$ (see Fig. 6a labeled "dark"). This indicates a temperature driven growth and generation of spherical Ag-nanoparticles within the PEI-layer respectively. Compared to suspensions in water the absorption peak is slightly red-shifted because of the different dielectric behavior of PEI. The samples irradiated at $70{ }^{\circ} \mathrm{C}$ show a comparable behavior to the suspensions in water. We found an increasing absorption peak at $\approx 550 \mathrm{~nm}$ with increasing irradiation time. As shown before, this indicates the growth of triangular and hexagonal silver nanoplates. On the other hand an absorption peak at $\approx 420 \mathrm{~nm}$ increases as well, even more than after pure heat treatment of the samples. Regarding the irradiated suspensions in water this peak decreased indicating a decrease in the number of spherical nanoparticles. The further growth of these particles within the PEI-layers during irradiation could be influenced by Ag-atom diffusion on the nanoparticle surface which is supposed to have a more prominent impact on the nanoparticle growth in the PEI than for the nanoparticle growth in a solvent like water.

In water the nanoparticle growth is dominated by Ag-ion diffusion to and adsorption at the excited regions at the irradiated nanoparticles. The ions get photoreduced at the excited regions leading to directed nanoparticle growth. As
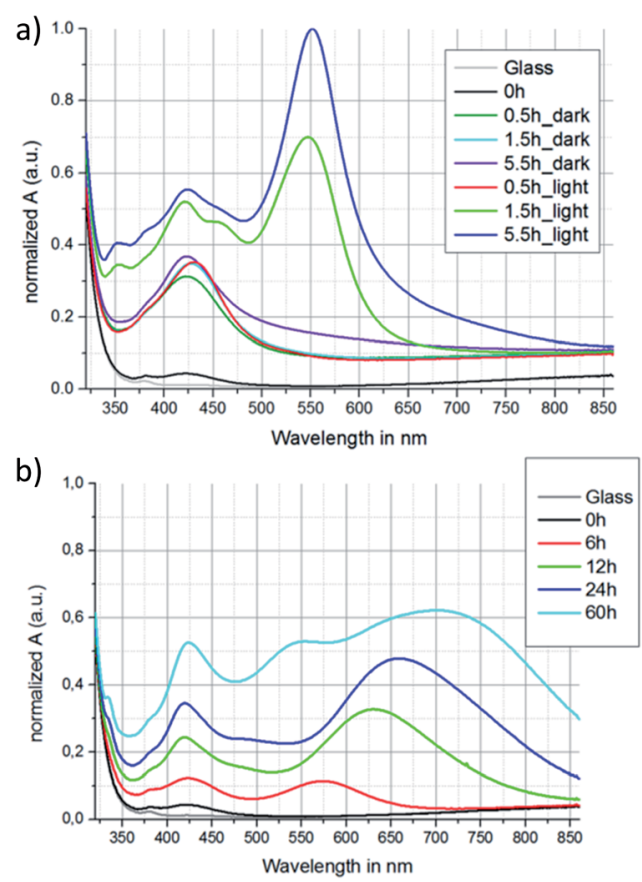

Fig. 6 Normalized absorption spectra of solution processed PEI thin films containing $5 \mathrm{mg} \mathrm{m}^{-1}$ silver nitrate. The different graphs correspond to different times of heat treatment at $70{ }^{\circ} \mathrm{C}$ in the dark (a), different irradiation times at $70^{\circ} \mathrm{C}(\mathrm{a})$, and different irradiation times at room temperature (b). a counterforce to this directed growing the reduced Ag-atoms start to migrate on the nanoparticle surface. Since the surface to volume ratio of the differently shaped nanoplates is larger than for nanospheres, the Ag-atoms will diffuse to positions at the particles corresponding to spherical shapes. However, the $\mathrm{Ag}$-atom diffusion at the surface of the nanoparticles is too slow to prevent the spherical nanoparticles from changing their shape. Therefore, the plate-like particles grow dominantly.

Within the PEI-layer the diffusion of Ag-ions is much slower leading to a slower formation rate of the nanoplates. Even though the Ag-ions preferably reduce at the excited regions at the nanoparticles the Ag-atoms will migrate at the particle surface resulting in the reshaping of the forming nanoplates. The absorption peak at $\approx 420 \mathrm{~nm}$ (spherical nanoparticles) and a much larger peak at $\approx 550 \mathrm{~nm}$ (hexagonal and triangular nanoplates) of the measured spectra indicate that the Ag-atom diffusion at the nanoparticle surface is slower than Ag-ion diffusion to the nanoparticles. Therefore, the growth of the nanoplates is still faster than the reshaping to nanospheres. However, the Ag-atom diffusion has a measurable impact on the nanoparticle shaping. Therefore, both nanoparticle types will grow leading to increasing absorption peaks at $\approx 420 \mathrm{~nm}$ and $\approx 550 \mathrm{~nm}$.

However, the elevated temperature still leads to a higher diffusion rate of the Ag-ions inside the PEI-layer, and therefore to an increased evolution speed of the hexagonal and triangular nanoplates compared to room temperature treatment. The very large absorption peak at $\approx 550 \mathrm{~nm}$ can be observed after only $5.5 \mathrm{~h}$ of constant irradiation (Fig. 6a). Longer irradiation periods lead to an increase of light absorption in the range of the whole measured spectra, and a color change of the films from light purple to grey occured. This indicates agglomeration of the nanoparticles also observable in water. ${ }^{25,26}$

Irradiation at room temperature also leads to a change in the absorption spectra of the layers, indicating a nanoparticle growth and reshaping as well (Fig. 6b). However, the diffusion of the Ag-ions within the PEI-layers is much slower than at $70^{\circ} \mathrm{C}$ leading to longer evolution time periods. For increasing irradiation time an increasing absorption peak at $\approx 420 \mathrm{~nm}$ can be observed again indicating the generation and growth of spherical AgNPs. A second peak increases with increasing irradiation time starting from $\approx 570 \mathrm{~nm}$ observed after $6 \mathrm{~h}$ but shifting to $\approx 700 \mathrm{~nm}$ after $60 \mathrm{~h}$. This behavior differs from the samples irradiated at $70{ }^{\circ} \mathrm{C}$. A reason could be the low Ag-ion diffusion rate respectively the low $\mathrm{Ag}$-seed generation rate at room temperature. Therefore, a smaller number of nanoparticles will consume more Ag-ions. This leads to a growth of a few big nanoplates instead of many smaller ones. We suggest the nanoparticle growth mechanism as schematically shown in Fig. 4b. Therefore, the growth of hexagonal and triangular plates of appropriate sizes for SPR at $\approx 550 \mathrm{~nm}$ is promoted for time periods up to $6 \mathrm{~h}$. However, with increasing time the particles get much bigger. Since the SPR of the particles depends on their size and shape ${ }^{42}$ the growth of the nanoplates leads to a shift of the absorption peak to longer wavelength. With increasing time periods the variety of different nanoparticle morphologies and sizes increases. Therefore, the 
absorption peaks of many differently sized and shaped particles overlap, leading to a broad absorption signal within the whole measured spectrum. Nevertheless, the prominent peaks at $\approx 420 \mathrm{~nm}$ and $\approx 700 \mathrm{~nm}$ indicate a preferred nanoparticle shape evolution, too.

\section{Concentration dependency}

To investigate the influence of the silver concentration on the nanoparticle evolution and the absorption properties of the PEIlayers, four different $\mathrm{Ag}$ concentrations were studied (see Table 1). All samples were irradiated at room temperature.

Fig. 7 shows the absorption spectra of the treated samples normalized to the absorption peak of $60 \mathrm{~h}$ irradiation treatment of sample D. The principle trend of all concentrations is comparable. With increasing time an absorption peak at $\approx 420 \mathrm{~nm}$ increases indicating the formation and growth of spherical silver nanoparticles again. With increasing concentration absorbance increases as well. After 60 hours of treatment the sample A shows $26 \%$ absorbance and sample B $46 \%$ absorbance compared to the peak value of sample D. These values are in good accordance to the respective concentration ratio and therefore indicate a proportional nanoparticle formation and growth rate for all investigated concentrations. However, sample C shows $84 \%$ absorbance compared to the peak value of sample $\mathrm{D}$. This might be caused by some inhomogeneity of the irradiation during the treatment. All samples show a shift of the increasing absorption peak starting at

Table 1 Silver concentration of different samples

\begin{tabular}{ll}
\hline Sample ID & Ag concentration in solution \\
\hline A & $5 \mathrm{mg} \mathrm{ml}^{-1}$ \\
B & $10 \mathrm{mg} \mathrm{ml}^{-1}$ \\
C & $15 \mathrm{mg} \mathrm{ml}^{-1}$ \\
D & $20 \mathrm{mg} \mathrm{ml}^{-1}$
\end{tabular}
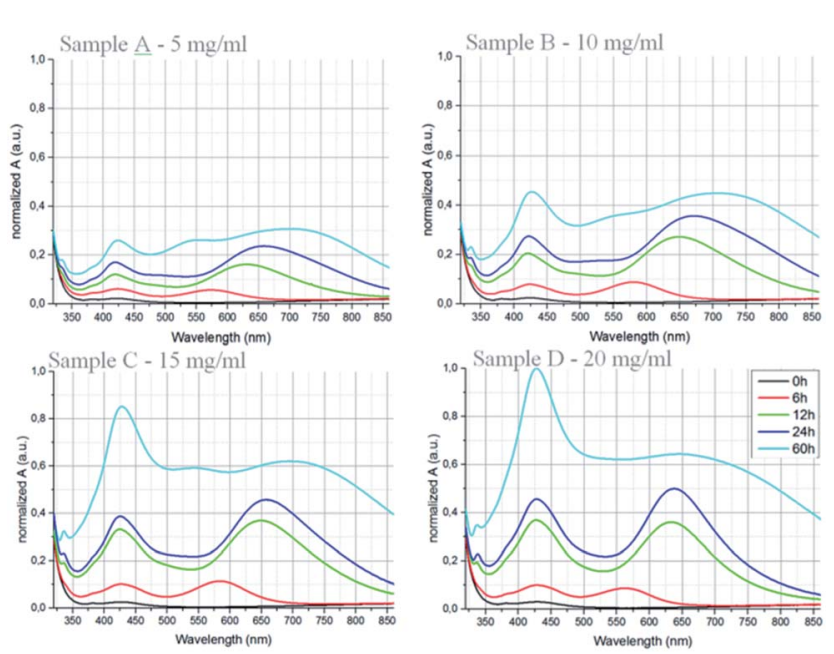

Fig. 7 Absorption spectra of the samples A, B, C and D treated for different time periods at room temperature. $\approx 570 \mathrm{~nm}$ after $6 \mathrm{~h}$ and shifting to $\approx 700 \mathrm{~nm}$ after $60 \mathrm{~h}$ of irradiation. This indicates a preferred nanoparticle morphology within these layers, too.

However, the absorption peak at $\approx 650 \mathrm{~nm}$ indicates different shapes and/or sizes compared to the previously found hexagonal and triangular plates. The spectra of sample C and D show more prominent peaks until $24 \mathrm{~h}$ of irradiation treatment than the samples A and B. The higher Ag-concentration and therefore the larger number of nanoparticles causes a faster nanoparticle growth. However, after $60 \mathrm{~h}$ the $420 \mathrm{~nm}$ absorbance peaks increased further whereas the $\approx 700 \mathrm{~nm}$ peak widens to an absorption band from $500 \mathrm{~nm}$ to $750 \mathrm{~nm}$ with a relative absorbance of $60 \%$ for both samples $C$ and D. This indicates an increasing morphology and/or size distribution of the nanoplates. This distribution leads to a variety of different SPR of the nanoplates within the optical wavelength range of $550 \mathrm{~nm}$ to $700 \mathrm{~nm}$.

For all samples a growing absorption peak at $\approx 550 \mathrm{~nm}$ can be observed after $60 \mathrm{~h}$ of constant irradiation treatment. This peak can be correlated to the triangular and hexagonal nanoplates again which also form during the irradiation at $70{ }^{\circ} \mathrm{C}$.

\section{Conclusions}

In this contribution we presented nanoparticle shaping within a polymer layer for the first time. Using this method we can control the morphology and therefore the optical properties of AgNPs within PEI-layers. The surface plasmon resonance of AgNPs embedded in this transparent polymer matrix is tailored by a nanoparticle evolution triggered by green light irradiation. The treatment temperature was found to play a significant role for the evolution of different nanoparticle shapes with high influence on the resulting absorption spectra and the necessary treatment time. The change in the equilibrium of Ag-atom diffusion rate at the nanoparticle surface and the Ag-ion diffusion rate inside the PEI thin films is suspected to be the reason for this impact. Furthermore, the concentration of Ag within the PEI-layers was found to influence the peak height of the increasing absorption peaks. The formed nanoparticle morphologies were observed by SEM-imaging. Different nanoparticle evolution states starting from spherical nanoparticles towards triangular and irregular and regular hexagonal nanoplates were observed in water suspensions. Because similar optical properties were observed within the PEI-layers we assume the same nanoparticle evolution process. These observations result in a novel hypothesis for the evolution of hexagonal and triangular shaped nanoplates. The SEM-images indicate that the growing nanoplates change their morphology from triangular to hexagonal and to larger triangles again.

To start the evolution within the PEI-layers we used two green LEDs operated at $200 \mathrm{~mA}$ for at least $1.5 \mathrm{~h}$ at $70{ }^{\circ} \mathrm{C}$. A possible application could be adaptable light filters for lab-on-achip fluorescent light detection. Lab-on-a-chip applications in the point of care market address short times and relatively low light intensities for measurement. The introduced routine clearly shows its potential to tailor optical filter properties for the use in these applications. 


\section{Conflicts of interest}

There are no conflicts to declare.

\section{Acknowledgements}

The work presented in this paper was funded by the German Federal Ministry of Education and Research (BMBF) within the joint research project "Handy-LoC" (FKZ 13N13717).

\section{References}

1 A. Alastalo, Technologies and Applications for Printed Conductors, 2008.

2 S. Molesa, D. R. Redinger, D. C. Huang and V. Subramanian, MRS Online Proc. Libr., 2003, 769, H8.3.

3 R. Venkata Krishna Rao, K. Venkata Abhinav, P. S. Karthik and S. P. Singh, RSC Adv., 2015, 5, 77760-77790.

4 M. L. Allen, Nanoparticle Sintering Methods and Applications for Printed Electronics, Aalto University, Helsinki, Finland, 2011.

5 K. G. Stamplecoskie, J. C. Scaiano, V. S. Tiwari and H. Anis, J. Phys. Chem. C, 2011, 115, 1403-1409.

6 A. Sánchez-González, S. Corni and B. Mennucci, J. Phys. Chem. C, 2011, 115, 5450-5460.

7 R. A. Sperling, Angew. Chem., Int. Ed., 2009, 48, 7289.

8 L. Mahmudin, E. Suharyadi, A. Bambang, S. Utomo and K. Abraha, J. Mod. Phys., 2015, 6, 1071-1076.

9 Y. Xia and N. J. Halas, MRS Bull., 2011, 30, 338-348.

10 J. Siegel, O. Kvítek, P. Ulbrich, Z. Kolská, P. Slepička and V. Švorčík, Mater. Lett., 2012, 89, 47-50.

11 D.-C. Tien, K.-H. Tseng, C.-Y. Liao, J.-C. Huang and T.-T. Tsung, J. Alloys Compd., 2008, 463, 408-411.

12 J. H. Jung, H. Cheol Oh, H. Soo Noh, J. H. Ji and S. Soo Kim, J. Aerosol Sci., 2006, 37, 1662-1670.

13 D. K. Lee and Y. S. Kang, ETRI J., 2004, 26, 252-256.

14 Q. Wang, S. T. Quek, C. T. Sun and X. Liu, Smart Mater. Struct., 2001, 10, 229.

15 C. M. Cobley, S. E. Skrabalak, D. J. Campbell and Y. Xia, Plasmonics, 2009, 4, 171-179.

16 M. Chen, Y. G. Feng, X. Wang, T. C. Li, J. Y. Zhang and D. J. Qian, Langmuir, 2007, 23, 5296-5304.

17 A. J. Christy and M. Umadevi, Adv. Nat. Sci.: Nanosci. Nanotechnol., 2012, 3, 35013-35014.

18 M. Sakamoto, M. Fujistuka and T. Majima, J. Photochem. Photobiol., C, 2009, 10, 33-56.

19 R. Sato-Berrú, R. Redón, A. Vázquez-Olmos and J. M. Saniger, J. Raman Spectrosc., 2009, 40, 376-380.

20 S. Kumar Ghosh, S. Kundu, M. Mandal, S. Nath and T. Pal, J. Nanopart. Res., 2003, 5, 577-587.

21 J. I. Hussain, Adv. Mater. Lett., 2011, 2, 188-194.
22 K. M. M. Abou El-Nour, A. Eftaiha, A. Al-Warthan and R. A. A. Ammar, Arabian J. Chem., 2010, 3, 135-140.

23 A. K. Suresh, D. A. Pelletier, W. Wang, J.-W. Moon, B. Gu, N. P. Mortensen, D. P. Allison, D. C. Joy, T. J. Phelps and M. J. Doktycz, Environ. Sci. Technol., 2010, 44, 5210-5215.

24 L. Sintubin, W. Verstraete and N. Boon, Biotechnol. Bioeng., 2012, 109, 2422-2436.

25 K. G. Stamplecoskie and J. C. Scaiano, J. Am. Chem. Soc., 2010, 132, 1825-1827.

26 M. Maillard, P. Huang and L. Brus, Nano Lett., 2003, 3, 16111615.

27 I. Pastoriza-Santos and L. M. Liz-Marzán, Langmuir, 1999, 15, 948-951.

28 Y. D. Sosa, M. Rabelero, M. E. Treviño, H. Saade and R. G. López, J. Nanomater., 2010, 2010, 1-6.

29 M. P. Neupane, S. J. Lee, I. S. Park, M. H. Lee, T. S. Bae, Y. Kuboki, M. Uo and F. Watari, J. Nanopart. Res., 2011, 13, 491-498.

30 C. A. dos Santos, A. F. Jozala, A. Pessoa and M. M. Seckler, J. Nanobiotechnol., 2012, 10(1), 43.

31 A. Shkilnyy, M. Soucé, P. Dubois, F. Warmont, M.-L. Saboungi and I. Chourpa, Analyst, 2009, 134, 1868.

32 W. Fritzsche, H. Porwol, A. Wiegand, S. Bornmann and J. M. Köhler, Nanostruct. Mater., 1998, 10, 89-97.

33 R. Nazar, S. Ronchetti, I. Roppolo, M. Sangermano and R. M. Bongiovanni, Macromol. Mater. Eng., 2015, 300, 226233.

34 H. Takele, H. Greve, C. Pochstein, V. Zaporojtchenko and F. Faupel, Nanotechnology, 2006, 17, 3499-3505.

35 G. V Ramesh, S. Porel and T. P. Radhakrishnan, Chem. Soc. Rev., 2009, 38, 2646.

36 Z. Shu, F. Kemper, E. Beckert, R. Eberhardt, A. Tünnermann, L. Edman, S. Inal, P. Pingel, K. Fostiropoulos, N. Koch, D. Neher and W. Ma, RSC Adv., 2017, 7, 26384-26391.

37 A. Pais, A. Banerjee, D. Klotzkin and I. Papautsky, Lab Chip, 2008, 8, 794-800.

38 A. Llobera, S. Demming, H. N. Joensson, J. Vila-Planas, H. Andersson-Svahn and S. Büttgenbach, Lab Chip, 2010, 10, 1987-1992.

39 C. Richard, A. Renaudin, V. Aimez and P. G. Charette, $L a b$ Chip, 2009, 9, 1371-1376.

40 J. G. Cuennet, A. E. Vasdekis and D. Psaltis, Lab Chip, 2013, 13, 2721-2726.

41 F. Kemper, E. Beckert, R. Eberhardt and A. Tünnermann, in Smart Systems Integration 2016-International Conference and Exhibition on Integration Issues of Miniaturized Systems, SSI 2016, 2016.

42 K. L. Kelly, E. Coronado, L. L. Zhao and G. C. Schatz, J. Phys. Chem. B, 2003, 107, 668-677. 